\title{
An analysis of preoperative delays prior to radical cystectomy for bladder cancer in Quebec
}

\author{
Nader Fahmy, MD; ${ }^{*}$ Wassim Kassouf, MD; ${ }^{*}$ Suganthiny Jeyaganth, $M S ;{ }^{*}$ Moamen Amin, MD; ${ }^{*}$ \\ Salaheddin Mahmud, MD; ${ }^{\dagger}$ Jordan Steinberg, MD; ${ }^{*}$ Simon Tanguay, MD; ${ }^{*}$ Armen Aprikian, MD*
}

See related article on page 109

\section{Abstract}

Background: The province of Quebec has the highest incidence of urothelial tumours in Canada. Radical cystectomy remains the standard treatment for invasive bladder cancer. We have previously observed that prolonged delays between transurethral resection of bladder tumour (TURBT) and radical cystectomy lead to worse survival in Quebec.

Objective: The aim of our study was to characterize the various periods of delay sustained by bladder cancer patients before radical cystectomy across Quebec and to determine their relation to survival.

Methods: We obtained the billing records for all patients treated with radical cystectomies for bladder cancer across Quebec from 1990 to 2002. Collected information included patient age and sex; dates of family physician (FP) and specialist visits with accompanying diagnoses; dates of cystoscopy, TURBT and CT scanning; surgeon age; surgical volume and dates of death.

Results: We analyzed a total of 25862 visits for 1633 patients. Median diagnostic delays from FP to specialist, then to cystoscopy, then to TURBT and finally from TURBT to CT were 20, 11, 4 and 14 days, respectively, over the entire study period. Median overall delay from FP visit to radical cystectomy was 93 days. In addition, median FP to radical cystectomy delay progressively increased from 1990 to 2000 from 58 to 120 days $(p<0.01)$. Multivariate analyses showed that patients with an overall delay of either $<25$ or $>84$ days had a 2.1 and 1.4 times increased risk of dying, respectively $(p \leq 0.01)$.

Conclusion: Preoperative delays have been progressively increasing over time. Overall, delays from FP to radical cystectomy of $<25$ and $>84$ days may translate into worse outcomes. Poor survival in cases with $<25$ days delay may be attributed to case selection, with more advanced cases being managed much quicker. Poor survival in cases with delays of $>84$ days may be attributed to disease progression while awaiting completion of management.

CUAJ 2008;2(2):102-8

\section{Introduction}

Bladder cancer is the sixth most common cancer in Canada, the eighth most common cause of cancer death in males and the thirteenth in females. ${ }^{1}$ About one-half of people with muscleinvasive bladder tumour have occult metastases at presentation, which reflects the aggressive nature of these tumours. ${ }^{2}$ Hence, it is preferable to avoid delays in definitive treatment once a diagnosis of invasive cancer has been made. Unfortunately, delays are sometimes unavoidable because of patient ${ }^{3,4}$ or health provider factors. ${ }^{5}$

Delays related to access to medical care have become a major concern in Canada. In Quebec, for all specialties combined, the median wait time from family physician (FP) referral until treatment more than doubled between 1993 and 2004, rising from 7 to 18 weeks. Median delays that are specifically related to urology have also increased by 5 weeks in 12 years. ${ }^{6}$ Several studies, including 1 from Quebec, have suggested that delays greater than 3 months between transurethral resection of bladder tumour (TURBT) and radical cystectomy were significantly associated with worse clinical outcome and mortality.,3,7-11 However, bladder cancer patients may be subjected to significant delays before TURBT for several reasons, including patients seeking additional advice, sick patients requiring medical optimization, delayed FP referral, delay in seeing a urologist and delay in obtaining investigations such as cystoscopy and imaging. To date, only a few publications included delays before TURBT in their outcome analyses. ${ }^{12-15}$ However, it is difficult to draw conclusions from these studies because of diverse study cohorts, different treatment protocols and lack of population perspective. Therefore, the 
objectives of our study were to characterize and measure the contribution of the different components of delay experienced by bladder cancer patients before radical cystectomy in Quebec, to identify predictors of longer delays and to determine the impact of the various components of delay on mortality.

\section{Methods}

\section{Study population}

We obtained complete billing records for 1633 patients who underwent radical cystectomy for bladder cancer in Quebec between 1990 and 2002 from the Quebec provincial database of physician fee-for-service claims (Régie de l'Assurance Maladie du Québec). Data received included patient age and sex, patient vital status and date of death, dates of FP and specialist visits, diagnoses coded using the International Classification of Diseases (ICD-9-CM) in each visit, dates of relevant diagnostic and therapeutic procedures (cystoscopy, TURBT, intravesical therapy, CT and radical cystectomy), anonymous unique identifier, age group of the urologist who provided the service and hospital name. The data set excluded patients aged less than 18 years and those preceded by any neoadjuvant therapy.

We measured several components of delay for each patient. Period 1 (FP or referral delay) is defined as the period from the first FP (office or emergency) visit where a diagnostic code associated with a bladder tumour (e.g., hematuria, cystitis) was recognized, until the first urologist visit. Period 2 (cystoscopy delay) is the period from the first urologist visit until the first cystoscopy. Period 3 (TURBT delay) is the period from the most recent cystoscopy until the first TURBT. Period 4 (imaging delay) is the period from the most recent TURBT until the date of CT imaging. To allow comparison with other reports we also examined the following: period 5 (diagnostic delay), defined as the period from the first urologist visit to $\mathrm{CT}$ imaging; period 6 (radical cystectomy delay), defined as the period from the first cystoscopy to the radical cystectomy; and period 7 (overall urologist delay), defined as the period from the first urologist visit to the radical cystectomy. We then integrated all delay periods together and examined period 8 (overall delay), defined as the period from the first FP visit to the radical cystectomy (Fig. 1).

For some patients, visits did not follow the chronological order shown in Figure 1. These visits were excluded from the calculation of their respective delays. For example, patients who had a urologist visit before their FP visit were excluded from the period 1 (FP or referral) delay analysis.

To minimize the effects of outliers (patients with unusually long delays), we excluded patients in whom delays were longer than the upper quartile of the preoperative delay period plus 3 times the interquartile range. ${ }^{16}$ Because we lacked information on tumour stage, patients who had more than 1 TURBT more than 4 months apart were classified as having a history of superficial tumours. All patients who had a history of superficial disease according to this criterion were excluded from the analysis of delay periods 5 to 8 .

\section{Statistical analyses}

Results were expressed as means and standard deviations (SDs). We first conducted both graphical (box plots) and statistical univariate analyses (Kruskall-Wallis test) to assess for the relationship between each delay variable and the following characteristics: patient sex, patient age, presence of hematuria, hospital radical cystectomy volume, surgeon radical cystectomy volume, surgeon age groups, and year, season and month of the tested procedure (cystoscopy, TURBT, CT or radical cystectomy). Using Cox regression and a stepwise modelling approach, variables with independent prognostic significance in relation to delay were identified and hazard ratios were generated for each variable.

To calculate overall survival in relation to delay,

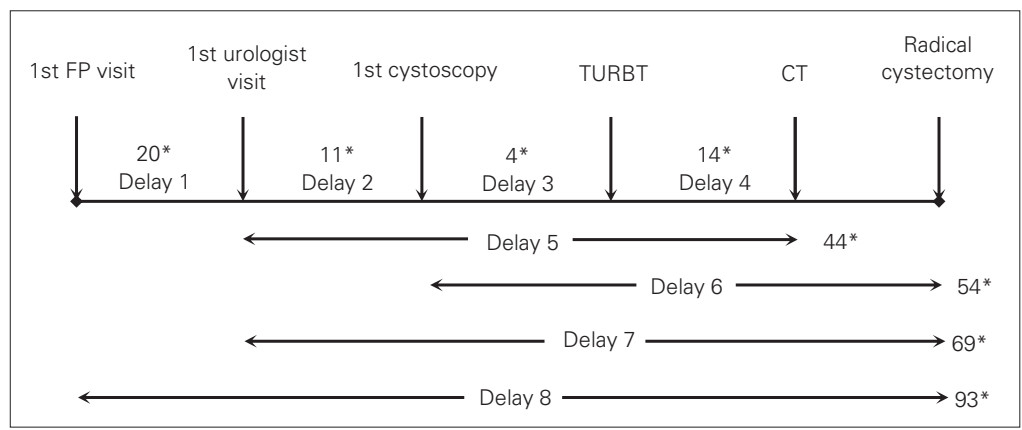

Fig. 1. Types of delays described and their median. FP = family physician; TURBT = transurethral resection of bladder tumour. *Median delay in days. 
we excluded patients who died within 90 days after radical cystectomy since these patients are

\begin{tabular}{|c|c|c|c|}
\hline Variable & $\begin{array}{c}\text { No. of patients, } \\
n=866\end{array}$ & $\begin{array}{l}\text { Median } \\
\text { delay, d } \\
(95 \% \mathrm{Cl})\end{array}$ & $\begin{array}{c}p \text { value } \\
\text { (Kruskal- } \\
\text { Wallis test) }\end{array}$ \\
\hline \multicolumn{4}{|c|}{ Period 1 (FP to urologist) } \\
\hline \multicolumn{4}{|l|}{ Sex } \\
\hline Female & 273 & $29(23-34)$ & $<0.001$ \\
\hline Male & 593 & $18(15-21)$ & \\
\hline \multicolumn{4}{|l|}{ Hematuria } \\
\hline No & 399 & 21 (19-24) & $<0.001$ \\
\hline Yes & 467 & $10(4-20)$ & \\
\hline \multicolumn{4}{|c|}{ Year of specialist visit } \\
\hline 1990-1995 & 376 & $18(14-21)$ & 0.008 \\
\hline 1996-2002 & 490 & $23(20-28)$ & \\
\hline \multicolumn{4}{|c|}{$\begin{array}{l}\text { Period } 3 \\
\text { (cystoscopy to TURBT) }\end{array}$} \\
\hline \multicolumn{4}{|l|}{ Sex } \\
\hline Female & 369 & $4(3-6)$ & 0.6 \\
\hline Male & 930 & $4(3-5)$ & \\
\hline \multicolumn{4}{|l|}{ Hematuria } \\
\hline No & 94 & $4(3-5)$ & 0.25 \\
\hline Yes & 1205 & $2(1-5)$ & \\
\hline \multicolumn{4}{|l|}{ Year of TURBT } \\
\hline 1990-1995 & 577 & $1(1-1)$ & $<0.001$ \\
\hline 1996-2002 & 722 & $8(7-8)$ & \\
\hline \multicolumn{4}{|c|}{ Period 4 (TURBT to CT) } \\
\hline \multicolumn{4}{|l|}{ Sex } \\
\hline Female & 323 & $11(4-35)$ & 0.12 \\
\hline Male & 757 & $15(4-36)$ & \\
\hline \multicolumn{4}{|l|}{ Hematuria } \\
\hline No & 68 & $14(4-36)$ & 0.22 \\
\hline Yes & 1012 & $8(2-37.5)$ & \\
\hline \multicolumn{4}{|l|}{ Year of CT } \\
\hline 1990-1995 & 504 & $8(4-27.5)$ & 0.01 \\
\hline 1996-2002 & 576 & $19(4-42)$ & \\
\hline \multicolumn{4}{|c|}{$\begin{array}{l}\text { Period } 8 \text { (FP to radical } \\
\text { cystectomy) }\end{array}$} \\
\hline \multicolumn{4}{|l|}{ Sex } \\
\hline Female & 209 & $96(82-117)$ & 0.81 \\
\hline Male & 452 & 92 (84-104) & \\
\hline \multicolumn{4}{|l|}{ Hematuria } \\
\hline No & 214 & 79 (62-93) & 0.002 \\
\hline Yes & 447 & 101 (91-114) & \\
\hline \multicolumn{4}{|l|}{$\begin{array}{l}\text { Year of radical } \\
\text { cystectomy }\end{array}$} \\
\hline 1990-1995 & 307 & 75 (62-84) & $<0.001$ \\
\hline 1996-2002 & 354 & 115 (104-129) & \\
\hline
\end{tabular}

more likely to have died because of operative complications. We then examined the association between various delay variables and overall survival computed from the date of the first FP visit to either the date of death or the date of censoring (Dec. 31, 2003). We used the Cox proportional hazards model with the shared frailty to assess the effect of preoperative delays on long-term survival. Models with shared frailty were used to allow for within-group correlation (among patients treated by the same surgeon). We assessed the assumption of proportional hazards for each of the covariates by examining graphs of scaled Schoenfeld residuals and by using appropriate statistical tests. All models were estimated using Stata 9 (Stata Corp., College Station, Texas).

\section{Results}

\section{Patient characteristics}

The mean patient age was 66.4 (SD 10.1) years and the median age was 69 years. Male to female ratio was 2.6:1. A total of 25862 visits were analyzed. Of the 1633 patients who had a radical cystectomy for bladder cancer included in this study, billing records revealed that $1288(78.9 \%)$ patients had FP visits, 1360 (83.3\%) had urologist visits, $1169(71.6 \%)$ had CT and $1516(92.8 \%)$ had TURBT. The overall 5 -year survival rate was $51 \%$. Table 1 illustrates the relation of various parameters to individual preoperative periods in univariate analysis.

\section{Variables affecting each delay type}

\section{Period 1 (FP to urologist)}

The number of patients who had complete information allowing for calculation of this period was 866. The median delay was 20 days (range 0-231 d). In the multivariate analysis, the odds of having delays shorter than 20 days were significantly associated with male sex (hazard ratio [HR] 0.65, 95\% confidence interval [Cl] 0.48-0.87, $p=0.005)$, or with the presence of hematuria (HR 0.55, 95\% Cl 0.34-0.75, $p<0.001$ ). Patients seen by a urologist after 1995 were significantly associated with longer delays (HR 1.38, 95\% Cl 1.05-1.83, $p=0.02$ ). 


\section{Period 2 (urologist to cystoscopy)}

The number of patients who had complete information for this period was 720 . The median delay was 11 days (range $0-128 \mathrm{~d}$ ). This delay had no correlation with any patient or provider characteristics.

\section{Period 3 (cystoscopy to TURBT)}

The number of patients who had complete information for this period was 1299. The median delay was 4 days (range 1-57 d). Multivariate analyses showed that the odds of having greater delays significantly correlated with TURBT performed after 1995 (HR 4.03, 95\% Cl 3.17-5.14, $p<0.001$ ).

\section{Period 4 (TURBT to CT imaging)}

The number of patients who had complete information for this period was 1080 . The median delay was 14 days (range 1-189 d). Multivariate analyses revealed that delays longer than 14 days were significantly associated with CT performed after 1995 (HR 1.95, 95\% Cl 1.44-2.64, $p<0.001$ ).

\section{Periods 5, 6 and 7}

These periods had no independent correlation with any variable. However, it was observed that these median delays significantly increased with time (Fig. 2).

\section{Period 8 (FP to radical cystectomy)}

The number of patients who had complete information for this period was 661. The median delay was 93 days (range 11-307). Multivariate analysis showed that delays shorter than 93 days were significantly associated with male sex (HR 0.65, $95 \% \mathrm{Cl} 0.48-0.87, p=0.005)$, the presence of hematuria (HR 0.50, 95\% Cl 0.34-0.75, $p<0.001$ ) or urologists aged $>59$ years (HR $0.25,95 \% \mathrm{Cl}$ $0.09-0.65, p=0.005)$. Radical cystectomies performed after 1995 were significantly associated with increased risks of been delayed more than 92 days (HR 1.38, 95\% Cl 1.05-1.83, $p=0.022$ ).

\section{Overall survival}

Period 8 and overall survival $(n=491)$ are shown in

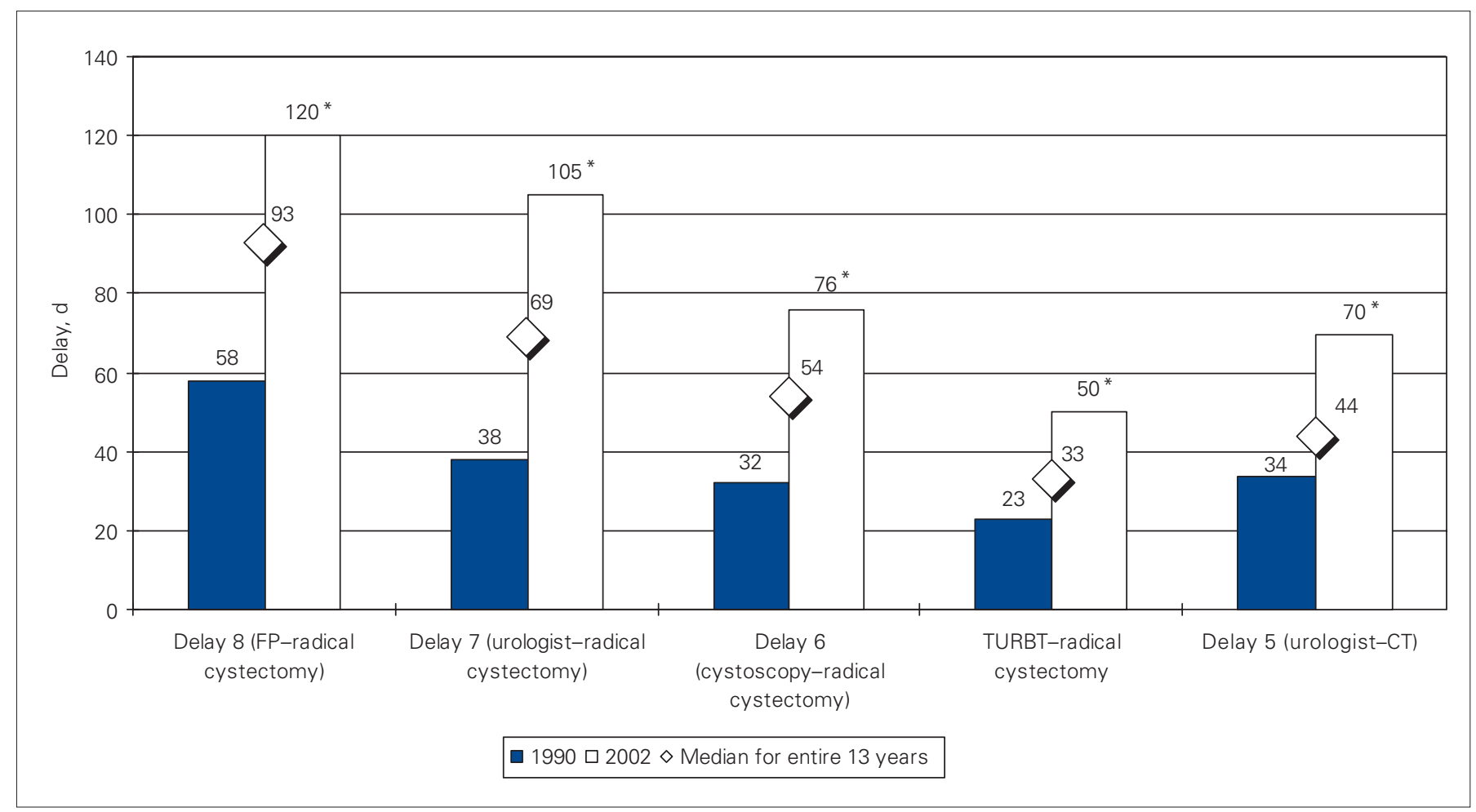

Fig. 2. Median delays investigated. $\mathrm{FP}=$ family physician. ${ }^{*} p=0.01$. 
Figure 3. Multivariate survival analyses have shown a significantly increased risk of overall mortality associated with patients who were aged $>69$ years (HR 2.3, 95\% Cl 1.4-3.9, $p=0.002$ ), patients who were delayed $<25$ days (HR 2.1,95\% Cl 1.3-3.3, $p=0.001)$ or those who were delayed $>84$ days (HR 1.4, 95\% Cl 1.1-1.8, $p=0.014)$.

\section{Discussion}

Since invasive bladder tumours are known to possess an aggressive nature from the time of presentation, it is expected that delaying definitive therapy might lead to worse outcome. Only a few publications included delays before TURBT in their outcome analyses. ${ }^{12-15}$ These publications had important variations, including diverse studied cohorts, inconsistent durations and types of delays, variable treatments, dissimilar investigated end points and different statistical methods used. Moreover, these studies were not conducted in Canada.

Our study has demonstrated that bladder cancer pretreatment delays have significantly increased over time in Quebec. A particularly long delay appears to occur between the FP visit and the urologist visit. The fact that younger age, female sex and absence of hematuria significantly correlated with longer delays could be in part due to the failure of the FP to appreciate the seriousness of the presenting symptom. Additionally, these delays might also occur in part because of difficulty in getting a timely urologist appointment. The second longest delay appears to be associated with prolonged CT scheduling that may be in part because of limited access to radiological resources.

Our analysis revealed that various delays were not associated with surgeon volume, surgeon age, hospital volume, the number of TURBTs, intravesical therapy, the season and the month of the procedure, or the type of diversion. In contrast, Hautmann and Paiss ${ }^{17}$ have shown that orthotopic bladders were significantly associated with shorter delays compared with ileal conduits.

Interestingly, ours is the first publication that studied the outcomes of delays from the FP visit

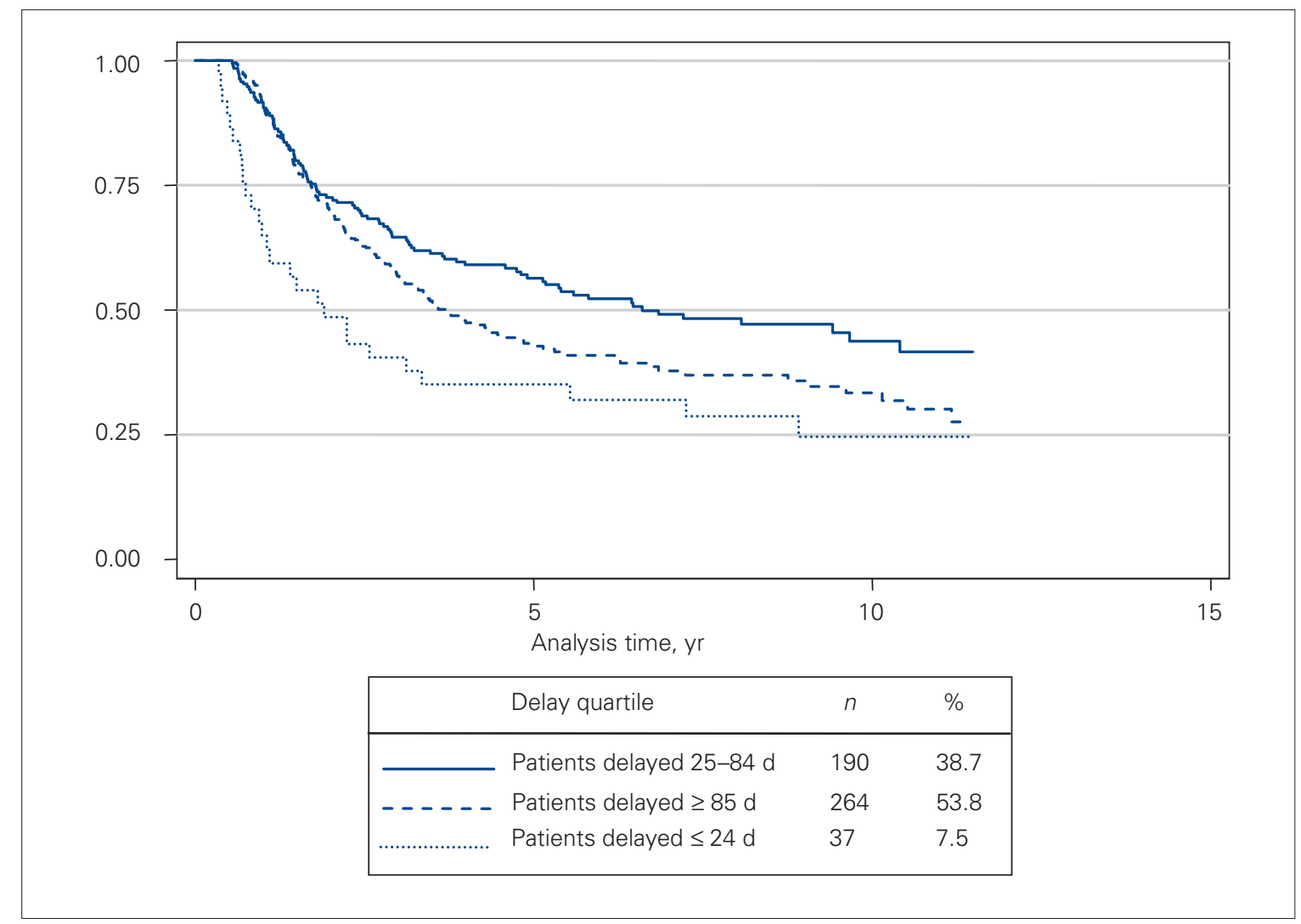

Fig. 3. Kaplan-Meier curves showing age adjusted overall survival of patients in relation to delays. $p=0.014$. 
until radical cytectomy that is not preceded by any neoadjuvant therapy. Few publications have investigated survival in relation to delays beyond the TURBT to radical cystectomy interval ${ }^{12-15}$ with significant variations among the reported delays, rendering comparison very difficult. Delays from the onset of symptoms to TURBT ${ }^{15}$ or to radiotherapy followed by radical cystectomy ${ }^{14}$ were not associated with a survival effect. These publications included patient delays that were based entirely on subjective assumptions. On the other hand, shorter delays from the first FP referral or first hospital appointment to TURBT ${ }^{13,15}$ or to radical cystectomy ${ }^{12,18}$ were associated with worse survival in some studies. The seemingly paradoxical worse survival associated with shorter delays was also noticed in our study where patients delayed $\leq 24$ days had higher mortality. This could be explained in part by a selection bias, where patients with higher-stage disease could have been expedited to receive faster treatment and ultimately died earlier. Conversely, we also observed significantly worse survival in patients delayed $>84$ days ( $12 \mathrm{wk}$ ). This observation may represent a true effect of disease progression while waiting for definitive therapy.

Causes for delays were reported in several studies $^{3,4,9,10,14,19}$ and ranged from patient to provider factors. Some of these delays can be reduced by increasing patients' awareness about early symptoms of bladder cancer, such as hematuria; by stressing the importance of early referral by FPs, especially for elderly female patients with hematuria, persistent cystitis, or both ${ }^{14,19,20}$; by initiating hematuria clinics, which have been shown to reduce delays caused by patient and FP factors ${ }^{20-23}$; and by addressing organizational and resource issues behind system or provider delays, or both. $., 20,24$

Finally, it should be noted that delay is not the only prognostic factor. There are other important predictors of survival, including clinical and surgical factors that could not be evaluated in our study.

This was a retrospective study that was based entirely on billing records that lacked information on disease-specific survival, disease stage and grade. Our study also cannot be accurately used to assess the existence of comorbidities. In addition, many of the provided visits were not valid for delay interpretation. The major strengths of our study lay in its provincial coverage and relatively large number of cases.

\section{Conclusion}

Bladder cancer pretreatment delays in Quebec have progressively increased over time. Younger age, female sex, the absence of hematuria and cystectomies performed after 1995 were significantly associated with longer delays between the FP visit and the radical cystectomy. Even though it is wise to encourage treatment as soon as the diagnosis of invasive bladder cancer is reached, this is unfortunately not always possible because of several factors including patient- or health care provider-related issues. Delays of more than 84 days between FP visit and radical cystectomy were significantly associated with a $40 \%$ increased risk of dying.

From the *Department of Surgery (Urology), McGill University, Montréal, Que., and the †Department of Community Heath Sciences, University of Manitoba, Winnipeg, Man.

This article has been peer reviewed.

Competing interests: None declared.

\section{References}

1. Canadian Cancer Society; National Cancer Insitiute of Canada; Statistics Canada. Canadian cancer statistics. Toronto; 2005.

2. Raghavan D, Quinn D, Skinner DG, et al. Surgery and adjunctive chemotherapy for invasive bladder cancer. Surg Oncol 2002;11:55-63.

3. May M, Nitzke T, Helke C, et al. Significance of the time period between diagnosis of muscle invasion and radical cystectomy with regard to the prognosis of transitional cell carcinoma of the urothelium in the bladder. Scand I Urol Nephrol 2004;38:231-5.

4. Sanchez-Ortiz RF, Huang WC, Mick R, et al. An interval longer than 12 weeks between the diagnosis of muscle invasion and cystectomy is associated with worse outcome in bladder carcinoma. J Urol 2003;169:110-5.

5. Simunovic M, Gagliardi A, McCready D, et al. A snapshot of waiting times for cancer surgery provided by surgeons affiliated with regional cancer centres in Ontario. CMAJ 2001;165:421-5.

6. Esmail N, Walker M. Waiting your turn: hospital waiting lists in Canada. Vancouver: The Fraser Institute; 2004.

7. Chang SS, Hassan JM, Cookson MS, et al. Delaying radical cystectomy for muscle invasive bladder cancer results in worse pathological stage. J Urol 2003;170:1085-7.

8. Hara I, Miyake H, Hara S, et al. Optimal timing of radical cystectomy for patients with invasive transitional cell carcinoma of the bladder. Jpn I Clin Oncol 2002;32:14-8.

9. Mahmud SM, Fong B, Fahmy N, et al. Effect of preoperative delay on survival in patients with bladder cancer undergoing cystectomy in quebec: a population based study. J Urol 2006; 175:78-83.

10. Lee CT, Madii R, Daignault S, et al. Cystectomy delay more than 3 months from initial bladder cancer diagnosis results in decreased disease specific and overall survival. J Urol 2006;175:1262-7.

11. Fahmy NM, Mahmud S, Aprikian AG. Delay in the surgical treatment of bladder cancer and survival: systematic review of the literature. Eur Urol 2006;50:1176-82.

12. Chahal R, Sundaram SK, Iddenden R, et al. A study of the morbidity, mortality and long- 
term survival following radical cystectomy and radical radiotherapy in the treatment of invasive bladder cancer in Yorkshire. Eur Urol 2003;43:246-57.

13. Gulliford MC, Petruckevitch A, Burney PG. Survival with bladder cancer, evaluation of delay in treatment, type of surgeon, and modality of treatment. BMJ 1991;303:437-40.

14. Mommsen S, Aagaard J, Sell A. Presenting symptoms, treatment delay and survival in bladder cancer. Scand J Urol Nephrol 1983;17:163-7.

15. Wallace DM, Bryan RT, Dunn JA, et al. Delay and survival in bladder cancer. BJU Int 2002;89:868-78.

16. Osborne JW, Overbay A. The power of outliers (and why researchers should always check for them). Practical assessment, research \& evaluation 2004;9(6). Available: http://PAREonline.net/getvn.asp?v=9\&n=6 (accessed 2008 Mar 2).

17. Hautmann RE, Paiss T. Does the option of the ileal neobladder stimulate patient and physician decision toward earlier cystectomy? J Urol 1998;159:1845-50.

18. Chahal R, Harrison SC. Re: an interval longer than 12 weeks between the diagnosis of muscle invasion and cystectomy is associated with worse outcome in bladder carcinoma. J Urol 2003:170:1327.
19. Wallace DM, Harris DL. Delay in treating bladder tumours. Lancet 1965;19:332-4.

20. Dickinson AJ, Howe K, Bedford C, et al. A retrospective study of the investigation and management of muscle-invasive bladder cancer in the South West Region. Br J Urol 1996;77:70-5.

21. Stower MJ. Delays in diagnosing and treating bladder cancer. Br Med I (Clin Res Ed) 1988:296:1228-9

22. Turner $A G$, Hendry WF, Williams $G B$, et al. A haematuria diagnostic service. BMJ 1977;2:29-31.

23. Paul AB, Collie DA, Wild SR, et al. An integrated haematuria clinic. Br I Clin Pract 1993; $47: 128-30$

24. Bishop MC. The dangers of a long urological waiting list. Br J Urol 1990;65:433-40.

Correspondence: Dr. Armen Aprikian, Head, Division of Urology, McGill University, Montreal General Hospital, L8-309, 1650 Cedar Ave., Montréal QC H3G 1A4; armen.aprikian@muhc.mcgill.ca

\title{
We welcome your comments on the journal and on specific articles.
}

\author{
All letters will be considered \\ for publication in the journal.
}

\section{Send your letters to the Editor-in-Chief at journal@cua.org}

CUAJ JAUC

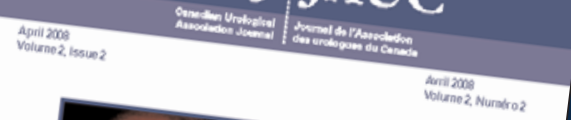
lumbotomy to repair

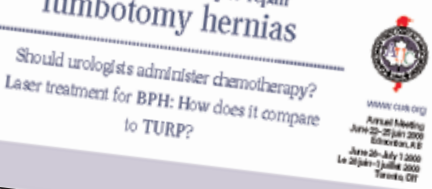

